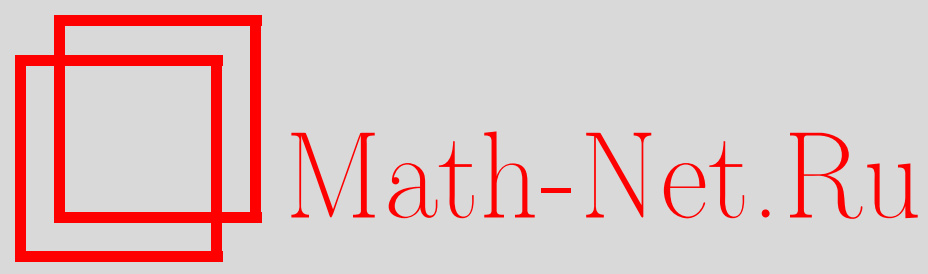

И. М. Романишин, Л. А. Синицкий, Об аддитивной $D$ устойчивости матрицы на основе критерия Харитонова, Матем. заметки, 2002, том 72, выпуск 2, 265-268

DOI: https://doi.org/10.4213/mzm420

Использование Общероссийского математического портала Math-Net.Ru подразумевает, что вы прочитали и согласны с пользовательским соглашением http://www.mathnet.ru/rus/agreement

Параметры загрузки:

IP: 34.229 .45 .116

26 апреля 2023 г., $17: 38: 33$

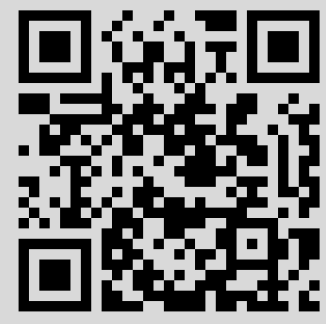




\title{
ОБ АДДИТИВНОЙ $D$-УСТОЙЧИВОСТИ МАТРИЦЫ НА ОСНОВЕ КРИТЕРИЯ ХАРИТОНОВА
}

\author{
И. М. Романишин, Л. А. Синицкий
}

На основе теоремы Харитонова приведены достаточные условия, которым должна удовлетворять $(n \times n)$-мерная матрица $A$ для того, чтобы $A-\operatorname{diag}\left(d_{1}, d_{2}, \ldots, d_{n}\right)$ была устойчивой при произвольных $d_{i} \geqslant 0, i=\overline{1, n}$.

Библиографоия: 10 названий.

На основе теоремы Харитонова [1] приведены достаточные условия, которым должна удовлетворять $(n \times n)$-мерная матрица $A$ для того, чтобы $A-D\left(D=\operatorname{diag}\left(d_{1}, d_{2}, \ldots, d_{n}\right)\right)$ была устойчивой при произвольных $d_{i} \geqslant 0, i=\overline{1, n}$.

Матрицу $A$ и ее характеристический полином будем назьвать устойчивы.ми (nолуустойчивым ми), если все собственные значения матрицы $A$, т.е. нули характеристического полинома, имеют отрицательные (неположительные) действительные части.

ОПредЕЛЕние [2]. Матрица $A$ назьвается аддитивно $D$-устойчивой, если $A-D$ $\left(D=\operatorname{diag}\left(d_{1}, d_{2}, \ldots, d_{n}\right)\right)$ устойчива при произвольных $d_{i} \geqslant 0, i=\overline{1, n}$.

$\mathrm{C}$ точки зрения теории цепей (в случае математической модели линейной цепи в виде системы дифференциальных уравнений в нормальной форме) принадлежность матрицы $A$ к классу аддитивно $D$-устойчивых матриц означает, что цепь остается устойчивой независимо от величины потерь в реактивных элементах, т.е. при включении последовательно с индуктивностями и параллельно емкостям произвольных резисторов с положительными сопротивлениями.

В теории систем управления аддитивная $D$-устойчивость означает устойчивость системы с отрищательной обратной связью с произвольным коэффициентом усиления [3]. Понятие аддитивной $D$-устойчивости находит также свое применение в задачах абсолютной устойчивости, при решении проблемы Айзермана [4], а также при исследовании нейронных цепей [5], в задачах эконометрии и математической экологии [6].

Подклассом аддитивно $D$-устойчивых матриц являются матрицы, для которых матричное уравнение Ляпунова имеет решение в виде диагональной матрицы с положительными элементами (в литературе такие матрицы называют D-дисcunamuвными [6], Lyapunov diagonally stable [2]). Рассмотрению таких матриц посвящена работа [7] и др.

Частньм случаем аддитивно $D$-устойчивых матриц являются матрицы, которые остаются устойчивыми при вычитании из одного или двух диагональных элементов произвольных неотрищательньх чисел. Условия, которьп должна удовлетворять $(n \times n)$ мерная матрица $A$ для того, чтобы $A-D\left(D=\operatorname{diag}\left(d_{1}, 0, \ldots, 0\right)\right)$ была устойчивой при 
произвольном $d_{1} \geqslant 0$, рассматривались в [3] на основе применения частотного критерия Найквиста, математического аппарата Лурье-Якубовича-Попова и др. В данной работе приведен критерий аддитивной $D$-устойчивости на основе применения теоремы Харитонова [1], который сводится к проверке устойчивости четырех полиномов Харитонова.

Известно [8], что характеристическое уравнение $(n \times n)$-мерной матрицы $A$ имеет вид

$$
\lambda^{n}+s_{1} \lambda^{n-1}+s_{2} \lambda^{n-2}+\cdots+s_{n}=0,
$$

где коэффициенты характеристического уравнения $s_{i}, i=\overline{1, n}$, являются суммами всех $(i \times i)$-мерных главных миноров матрицы $A$, умноженных на $(-1)^{i}$. Необходимым условием устойчивости матрицы $A$ является положительность коэффициентов характеристического уравнения $s_{i}, i=\overline{1, n}$.

Рассмотрим структуру коэффициентов характеристического уравнения для матрицы $A-D\left(D=\operatorname{diag}\left(d_{1}, d_{2}, \ldots, d_{n}\right), d_{i} \geqslant 0, i=\overline{1, n}\right)$. Для простоты понимания запишем характеристическое уравнение для $(3 \times 3)$-матрицы $A-D\left(D=\operatorname{diag}\left(d_{1}, d_{2}, d_{3}\right), d_{i} \geqslant 0\right.$, $i=1,2,3)$ :

$$
\begin{aligned}
\lambda^{3}+ & \lambda^{2}\left[-\left(a_{11}+a_{22}+a_{33}\right)+d_{1}+d_{2}+d_{3}\right] \\
+ & \lambda\left[A_{12}+A_{13}+A_{23}+d_{2} d_{3}+d_{1} d_{3}+d_{1} d_{2}\right. \\
& \left.-d_{1}\left(a_{22}+a_{33}\right)-d_{2}\left(a_{33}+a_{11}\right)-d_{3}\left(a_{11}+a_{22}\right)\right] \\
+ & {\left[-\operatorname{det} A+d_{1} d_{2} d_{3}-d_{1} d_{2} a_{33}-d_{1} d_{3} a_{22}-d_{2} d_{3} a_{11}\right.} \\
& \left.+d_{1} A_{23}+d_{2} A_{13}+d_{3} A_{12}\right]=0,
\end{aligned}
$$

где $a_{i i}$ - диагональные элементы матрищы $A, A_{i j}$ - главные миноры, включающие $i$-е и $j$-е строки и столбцы.

Можно показать, что для $(n \times n)$-матрищы $A-D\left(D=\operatorname{diag}\left(d_{1}, d_{2}, \ldots, d_{n}\right)\right)$ характеристическое уравнение запишется в виде

$$
\begin{aligned}
& \lambda^{n}+\left(s_{1}+\sum_{i=1}^{n} d_{i}\right) \lambda^{n-1}+\left(s_{2}+\sum_{i=1}^{n} \sum_{\substack{j=1 \\
j \neq i}}^{n} d_{i} d_{j}+(-1) \sum_{i=1}^{n} d_{i} B_{i}^{(1)}\right) \lambda^{n-2} \\
& +\left(s_{3}+\sum_{i=1}^{n} \sum_{\substack{j=1 \\
j \neq i}}^{n} \sum_{\substack{k=1, k \neq j \\
k \neq i}}^{n} d_{i} d_{j} d_{k}\right. \\
& \left.+(-1) \sum_{i=1}^{n} \sum_{\substack{j=1 \\
j \neq i}}^{n} d_{i} d_{j} B_{i j}^{(1)}+(-1)^{2} \sum_{i=1}^{n} d_{i} B_{i}^{(2)}\right) \lambda^{n-3}+\cdots \\
& +s_{n}+\prod_{i=1}^{n} d_{i}+(-1) \underbrace{\sum \cdots \sum}_{n-1} \underbrace{d_{i} d_{j} d_{k} \ldots}_{n-1} B_{n-1}^{B_{i j k \ldots}}+\cdots \\
& +(-1)^{p} \underbrace{\sum \cdots \sum}_{n-p} \underbrace{d_{i} d_{j} d_{k} \ldots}_{n-p} B_{\underbrace{(p)}_{n-p}}^{(p) \ldots}+\cdots+(-1)^{n-1} \sum_{i=1}^{n} d_{i} B_{i}^{(n-1)}=0,
\end{aligned}
$$

где $B_{n-p}^{(p)}-$ сумма всех $(p \times p)$-мерных главных миноров матрищы, которая получается из матрицы $A$ после вычеркивания $n-p$ строк и столбцов с номерами $i, j, k \ldots$ 
ОПРеДЕЛЕниЕ [9]. Матрица $A$ назьвается парииально устойчивой (парииально полуустойчивой), если все ее главные подматрицы устойчивы (полуустойчивы).

Необходимым условием аддитивной $D$-устойчивости является парциальная полуустойчивость. Легко проверить (например, при помощи критерия Рауса-Гурвища), что для $(3 \times 3)$-матрищы парциальной устойчивости достаточно для аддитивной $D$-устойчивости. Такой критерий не установлен для $(n \times n)$-матрищ, и вопрос эффективного критерия аддитивной $D$-устойчивости матриц в общем случае остается открытым. Одна из попыток его решения представлена здесь.

Главные миноры размера $i \times i$ парциально полуустойчивых матрищ, умноженные на $(-1)^{i}$, неотрицательны. Поэтому составляющие коэффищиентов характеристического уравнения (2) неотрицательны. Это означает, что коэффициенты характеристического уравнения (2) принимают минимальные значения при $d_{i}=0, i=\overline{1, n}$, а максимальныепри максимальных значениях $d_{i} \geqslant 0, i=\overline{1, n}$. Максимальные значения $d_{i}^{*} \geqslant 0, i=\overline{1, n}$, выбираем из условия принадлежности матрицы $A-D^{*}\left(D^{*}=\operatorname{diag}\left(d_{1}^{*}, d_{2}^{*}, \ldots, d_{n}^{*}\right)\right) \mathrm{k}$ матрицам с диагональньм преобладанием по строкам или столбцам, т.е.

$$
d_{i}^{*}=\sum_{\substack{j=1 \\ j \neq i}}^{n}\left|a_{i j}\right|+a_{i i}, \quad i=\overline{1, n}
$$

или

$$
d_{i}^{*}=\sum_{\substack{j=1 \\ j \neq i}}^{n}\left|a_{j i}\right|+a_{i i}, \quad i=\overline{1, n} .
$$

Для тех $i$, при которых выражения (3), (4) отрицательны, принимаем $d_{i}^{*}=0$. При $d_{i}>d_{i}^{*}, i=\overline{1, n}$, матрица $A-D$ устойчива. Таким образом, задача аддитивной $D$-устойчивости свелась к условиям устойчивости интервальной матрицы, точнее интервального полинома вида (1) при

$$
d_{i} \in\left[0, d_{i}^{*}\right], \quad d_{i}^{*} \geqslant 0, \quad i=\overline{1, n}
$$

и может быть эффективно решена на основе теоремы Харитонова.

Теорема. Для того чтобы матрица $A-D\left(D^{*}=\operatorname{diag}\left(d_{1}, d_{2}, \ldots, d_{n}\right)\right)$ была устойчивой при произвольных $d_{i} \geqslant 0, i=\overline{1, n}$, достаточно, чтоб были устойчивыми четыре характеристических полинома $R_{i}(\lambda), i=\overline{1,4}$ :

$$
\begin{aligned}
& R_{1}(\lambda)=s_{n}+s_{n-2}^{*} \lambda^{2}+s_{n-4} \lambda^{4}+s_{n-6}^{*} \lambda^{6}+\cdots+s_{n-1} \lambda+s_{n-3}^{*} \lambda^{3}+\cdots, \\
& R_{2}(\lambda)=s_{n}+s_{n-2}^{*} \lambda^{2}+s_{n-4} \lambda^{4}+s_{n-6}^{*} \lambda^{6}+\cdots+s_{n-1}^{*} \lambda+s_{n-3} \lambda^{3}+\cdots, \\
& R_{3}(\lambda)=s_{n}^{*}+s_{n-2} \lambda^{2}+s_{n-4}^{*} \lambda^{4}+s_{n-6} \lambda^{6}+\cdots+s_{n-1} \lambda+s_{n-3}^{*} \lambda^{3}+\cdots, \\
& R_{4}(\lambda)=s_{n}^{*}+s_{n-2} \lambda^{2}+s_{n-4}^{*} \lambda^{4}+s_{n-6} \lambda^{6}+\cdots+s_{n-1}^{*} \lambda+s_{n-3} \lambda^{3}+\cdots,
\end{aligned}
$$

әде $s_{i}, s_{i}^{*}, i=\overline{1, n},-$ коэффициенты характеристических уравнений для матрии, $A$ и $A-D^{*}$ соответственно. 
ДоКАЗАТЕЛЬСТвО. Устойчивость четырех характеристических полиномов Харитонова обеспечивает устойчивость матрицы $A-D$ при принадлежности $d_{i}$ интервалу (5), в том числе и при $d_{i}=d_{i}^{*}, i=\overline{1, n}$. На основе непрерывности собственных значений матрицы от ее элементов заключаем, что матрица $A-D$ устойчива в окрестности $d_{i}=d_{i}^{*}$, $i=\overline{1, n}$, т.е. при $d_{i} \in\left[d_{i}^{*}-\varepsilon, d_{i}^{*}+\varepsilon\right]$, где $\varepsilon>0$ - сколь угодно малое положительное число. При $d_{i}>d_{i}^{*}$ матрица $A-D$ является матрицей со строгим диагональным преобладанием по строкам или столбцам и, таким образом, устойчива в силу критерия Гершгорина [8].

Отметим, что критерий Харитонова [1] задает необходимые и достаточные условия устойчивости и интервальных полиномов. Однако приведенные условия аддитивной $D$-устойчивости матрищы на основе теоремы Харитонова являются только достаточными из-за того, что при изменении $d_{i}, i=\overline{1, n}$, коэффициенты характеристического полинома (2) не могут изменяться независимо, и имеются совместные ограничения на возмущения этих коэффициентов аналогично случаю эллиптических ограничений [10].

\section{СПИСОК ЦИТИРОВАННОЙ ЛИТЕРАТУРЫ}

[1] Харитонов В. Л. Об асимптотической устойчивости положения равновесия семейства систем линейных дифференциальных уравнений // Дифференц. уравнения. 1978. Т. 14. № 11. C. $2086-2088$.

[2] Hershkowitz D. Recent directions in matrix stability // Linear Algebra Appl. 1992. V. 171. P. 161-186.

[3] Романишин И. М., Синицкий Л. А. $H_{D}$-системы // Проблемы управления и информатики. 1996. Т. 1-2. С. 224-238.

[4] Романишин И. М., Синицкий Л. А. О некоторых классах матриц и проблеме Айзермана // Проблемы управления и информатики. 1998. Т. 6. С. 14-24.

[5] Kaszkurewicz E, Bhaya A. Comments on "Necessary and Sufficient Condition for Absolute Stability of Neural Networks" // IEEE Trans. on Circuits and System. 1995. V. 42. № 8 . P. 497-499.

[6] Пых Ю.А. Равновесие и устойчивость в моделях популяционной динамики. М.: Наука, 1983.

[7] Barker G. R., Berman A., Plemmons R. J. Positive diagonal solutions to the Lyapunov equations // Linear and Multilinear Algebra. 1978. V. 5. P. 249-256.

[8] Гантмахер Ф.Р. Теория матриц. М.: Наука, 1988.

[9] Синицкий Л. А. О свойствах матриц, применяемых в теории электрических цепей // Теоретическая электротехника. 1989. № 47. С. 39-45.

[10] Поляк Б. Т., Цыпкин Я. З. Частотные критерии робастной устойчивости и апериодичности линейных систем // Автоматика и телемеханика. 1990. № 9. С. 45-54.

(И.М. Романишин) Физико-механический институт им. Г. В. Карпенко НАН Украины Поступило (Л.А. Синицкий) Львовский государственный университет им. И. Франко

15.06.2001 